\section{Commentary on: Disinfection of dental impressions - compliance to accepted standards}

\author{
N. Almortadi and R. G. Chadwick
}

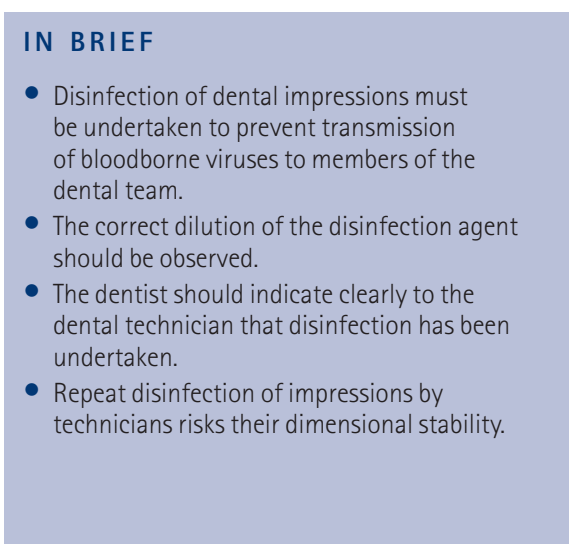

Introduction The responsibility of ensuring impressions have been cleaned and disinfected before dispatch to the dental laboratory lies solely with the dentist. Uncertainty of impression disinfection risks both the health of the receiving dental technician and potential repeat disinfection of an already disinfected impression with detrimental consequences for its dimensions. Objective To ascertain, from the perspectives of dentists and dental technicians, current impression decontamination and disinfection practices with, in the case of the technicians, an estimate of the relative prevalence of contaminated voids within apparently disinfected impressions. Design Anonymous postal questionnaire. Method Dentist $(n=200)$ and dental technician $(n=200)$ potential participants, selected at random from the registers held by the General Dental Council, were invited to complete an anonymous postal questionnaire that sought to establish current practices and perceived effectiveness of impression disinfection. Results Questionnaire return rates of $42.1 \%$ and $31.2 \%$ were recorded for dentists and dental technicians respectively. A wide range of solutions, at different dilutions of the same product, was used by the dentists to disinfect dental impressions. 37.2\% rinsed the impressions with water, and 2.6\% always brushed debris away, before disinfection. $24.7 \%$ of dentists did not inform the laboratory of disinfection. Irrespective of the disinfection status of the received impressions, 50\% of the responding dental technicians disinfected all impressions. 95\% of them had received blood-contaminated impressions. 15\% had encountered blood-filled voids upon trimming back the peripheries of impressions. 64.7\% were confident that the impressions received by them had been disinfected by the dentists. Conclusions Compliance with good practice is less than ideal and education in impression disinfection for both dentists and dental technicians is required to address this.

\section{COMMENTARY}

The BDA places the responsibility squarely on the shoulders of dentists to clean and disinfect impressions before sending them to the laboratory. Consequently, this is a very useful study as it surveys the practice of both dentists and technicians. A key finding was that whilst 95\% of responding dentists claim to disinfect impressions only 37\% first decontaminate the impression by rinsing. Not surprisingly, most technicians had received blood-stained impressions and many were unsure whether the dentist had in any case disinfected the impression or with what if they had. The upshot was half the responding laboratories routinely disinfected impressions irrespective of what had been written on the laboratory sheet.

The authors emphasise that repeated disinfection may cause dimensional errors or loss of surface detail. Although this hypothesis was not tested it is known that prolonged immersion in some disinfect- ants (more than one hour) may result in measurable changes to impression surface detail. Impression materials are variably sensitive to such changes, with some reversible hydrocolloids (alginates) being particularly so. Furthermore, any interaction between the plethora of disinfectants used by dentists and technicians remains unstudied but could be another potential source of cast error. For these reasons it is best to follow the BDA's and authors' advice to disinfect impressions reliably before leaving the surgery. However, dentists first need to convince technicians that they have dependable systems in place, which is why it is so important to have a two-way dialogue with laboratories to understand each other's point of view. Hopefully this study will promote such discussions.

The response rate to the questionnaire was less than ideal (42\% from dentists and $32 \%$ from technicians). Nevertheless, the authors found strong arguments to defend the validity of their findings. Other work- ers relying on questionnaires should bear in mind that these arguments are specific to this study and may not transfer readily to other studies. With dentists receiving so many requests to complete questionnaires researchers need to consider suitable inducements to optimise response.

The paper emphasises the importance of rinsing impressions after removal from a patient's mouth. It is also worth emphasising the importance of rinsing after disinfection to remove excess disinfectant which may have a deleterious effect on impression accuracy during storage. The advice to brush an impression whilst rinsing may have merit for elastomeric impressions but risks damaging alginate impressions, particularly if the brush has stiff bristles.

R. Wassell Clinical Senior Lecturer,

Restorative Dentistry, Newcastle University School of Dental Sciences DOI: 10.1038/sj.bdj.2010.1155 